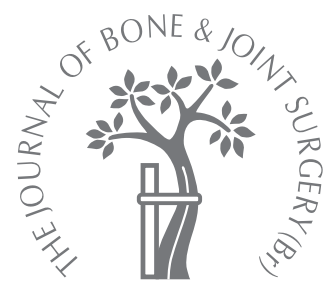

\title{
Does anterior cruciate ligament reconstruction restore normal knee kinematics?
}

\author{
A PROSPECTIVE MRI ANALYSIS OVER TWO YEARS
}

\begin{abstract}
J. M. Scarvell, P. N. Smith, K. M. Refshauge, H. R. Galloway, K. R. Woods
\end{abstract}

From The Canberra Hospital, Garran, Australia

\begin{abstract}
J. M. Scarvell, PhD,
Physiotherapist, University Lecturer

P. N. Smith, FRACS,

Associate Professor

Orthopaedic Surgery

Trauma and Orthopaedic

Research Unit

12A The Canberra Hospital, Yamba Drive, Garran ACT

2605, Australia.

K. M. Refshauge, PhD, Professor

School of Physiotherapy

University of Sydney, East

Street, Lidcombe, New

South Wales 2141, Australia.

H. R. Galloway, FRANZCR, Associate Professor

Radiology, Director Medical Imaging

ANU Medical School

Australian National

University, The Canberra

Hospital, Garran ACT 2605,

Australia.
\end{abstract}

K. R. Woods, FRACS

Orthopaedic Surgeon

Canberra Orthopaedic Group

John James Hospital, ACT

2600, Australia.

Correspondence should be sent to Dr J. M. Scarvell; e-mail: jennie.scarvell@ canberra.edu.au

(C2006 British Editorial Society of Bone and Joint Surgery doi:10.1302/0301-620X.88B3. $16787 \$ 2.00$

$J$ Bone Joint Surg [Br] 2006;88-B:324-30.

Received 31 May 2005 Accepted after revision 26 October 2005

This prospective study used magnetic resonance imaging to record sagittal plane tibiofemoral kinematics before and after anterior cruciate ligament reconstruction using autologous hamstring graft. Twenty patients with anterior cruciate ligament injuries, performed a closedchain leg-press while relaxed and against a $150 \mathrm{~N}$ load. The tibiofemoral contact patterns between $0^{\circ}$ to $90^{\circ}$ of knee flexion were recorded by magnetic resonance scans. All measurements were performed pre-operatively and repeated at 12 weeks and two years.

Following reconstruction there was a mean passive anterior laxity of $2.1 \mathrm{~mm}$ (SD 2.3), as measured using a KT 1000 arthrometer, and the mean Cincinnati score was 90 (SD 11) of 100. Pre-operatively, the medial and lateral contact patterns of the injured knees were located posteriorly on the tibial plateau compared with the healthy contralateral knees $(p=0.014)$, but were no longer different at 12 weeks $(p=0.117)$ or two years postoperatively $(p=0.909)$. However, both reconstructed and healthy contralateral knees showed altered kinematics over time. At two years, the contact pattern showed less posterior translation of the lateral femoral condyle during flexion $(p<0.01)$.

Anterior cruciate ligament (ACL) injury alters the kinematics of the knee and degenerative changes may follow. There is no good evidence that reconstruction prevents osteoarthritis, with a $7 \%$ to $18 \%$ incidence of osteoarthritis reported at seven years following bone-patellar tendon-bone reconstruction ${ }^{1,2}$ and a $4 \%$ to $16 \%$ incidence at five years following hamstring reconstruction. ${ }^{2,3}$ Longer term studies using contemporary ligament reconstruction techniques are not yet available.

Since the abnormal kinematics of the ACLdeficient knee have been considered partly responsible for a high incidence of late osteoarthritis, ${ }^{4}$ research has focused on the kinematics of the knee before and after ligament reconstruction..$^{5-9}$ In vitro studies suggest that while anterior stability of the reconstructed knee is similar to the normal knee, ${ }^{10,11}$ rotary stability is not restored by reconstruction. ${ }^{12}$ In contrast, the results of in vivo studies following hamstring reconstruction differ as to whether dynamic anterior translation ${ }^{13-16}$ or rotation are restored to normal. ${ }^{8}{ }^{8}$ In vivo studies using highly accurate but invasive roentgen stereophotogrammetric analysis (RSA) have shown that abnormalities of rotation persist following reconstruction..$^{8,9,17}$

Kinematics expressed by the tibiofemoral contact pattern following reconstruction have not been evaluated despite the importance of tibiofemoral interface events in the development of degenerative changes. Knees with acute ACL injuries have been shown to have different contact patterns to normal knees, particularly in the lateral compartment. ${ }^{18}$ In the chronic ACL-deficient knee, it is the medial compartment contact pattern that is affected, as the secondary restraints to anterior translation are attenuated by chronic loading. ${ }^{19}$ Whether these contact patterns are corrected by reconstructive surgery is not known.

A prospective study was designed using magnetic resonance imaging (MRI) to record the tibiofemoral contact pattern of the ACLinjured knees and normal contralateral knees of patients undergoing reconstruction using autologous hamstring grafts. Tibiofemoral contact was to be recorded while performing a closed-chain leg-press, pre-operatively and post-operatively at 12 weeks and at two years' follow-up, in order to determine whether reconstruction restores normal contact patterns.

\section{Patients and Methods}

We recruited 20 patients with a unilateral ACL injury. There were eight men and 12 women. The mean age of patients was 33 years (SD 7; 19 to 52). The injuries were sustained at a 
mean of seven months (SD 10; 1 to 36). There were 12 right knees injured and eight left knees. The injuries occurred during soccer (7), netball (4), basketball (2), skiing (2), martial arts (2), football (1), a motorbike accident (1) and a fall from $1 \mathrm{~m}$ height (1). All ACL injuries were diagnosed clinically by an orthopaedic surgeon and confirmed later at arthroscopy. Clinical and arthroscopic examination confirmed that no patients had any ligamentous damage in addition to the ACL injury. However, at arthroscopy three patients had trimming of the medial meniscus. This involved less than one third of the meniscus in all cases, and there were no meniscal repairs. Two patients also had early degenerative changes affecting the medial femoral condyle, which did not require treatment.

All patients were available for follow-up at 12 weeks post-operatively and 18 were available at two years. Of the two patients lost to follow-up, one had moved internationally and one had undergone further surgery with cartilage transplantation and was therefore excluded from the study. Patients were excluded if there were contraindications to MRI, if they may have been pregnant, if they were over 180 $\mathrm{cm}$ tall (to permit knee flexion in the MRI tunnel) or if there was a history of injury or symptoms in the contralateral knee.

We used 12 healthy patients aged between 20 and 50 years (seven men and five women) as controls for the comparison of knee kinematics at two years. None had any symptoms or history of injury in either knee. The kinematic findings in these patients have been reported previously. ${ }^{20}$

Ethical approval for the study was obtained from the University and Department of Health human research ethics committees and all patients gave informed consent. Operative procedure. Knee reconstruction was performed by four orthopaedic surgeons (PNS, KRW, MG, RC) using the same technique. The ACL was replaced using a fourstrand hamstring graft of semi-tendinosus and gracilis and the intraarticular part of the procedure was performed arthroscopically. Fixation was over a transverse femoral pin within the femoral tunnel proximally and by means of a bioabsorbable interference screw supplemented with two staples distally. Post-operative rehabilitation allowed early full weight-bearing as tolerated, followed by an accelerated regime with closed-chain exercises and proprioception training. ${ }^{21,22}$ Return to sport was permitted at six months. Outcomes. Passive anterior laxity was measured for each patient pre-operatively and at 12 weeks and two years postoperatively using a KT 1000 knee arthrometer (Medmetric, San Diego, California). The difference between the injured knee and healthy contralateral knee was recorded at $134 \mathrm{~N}$ of anterior drawer and maximum manual anterior drawer, according to the protocol established by Daniel, Malcolm and Losse. ${ }^{23}$

The Cincinnati knee rating system ${ }^{24}$ which takes into account the severity of symptoms and difficulties with daily living, as well as the examination and radiological findings, was also used. The best possible score is 100 . It has been

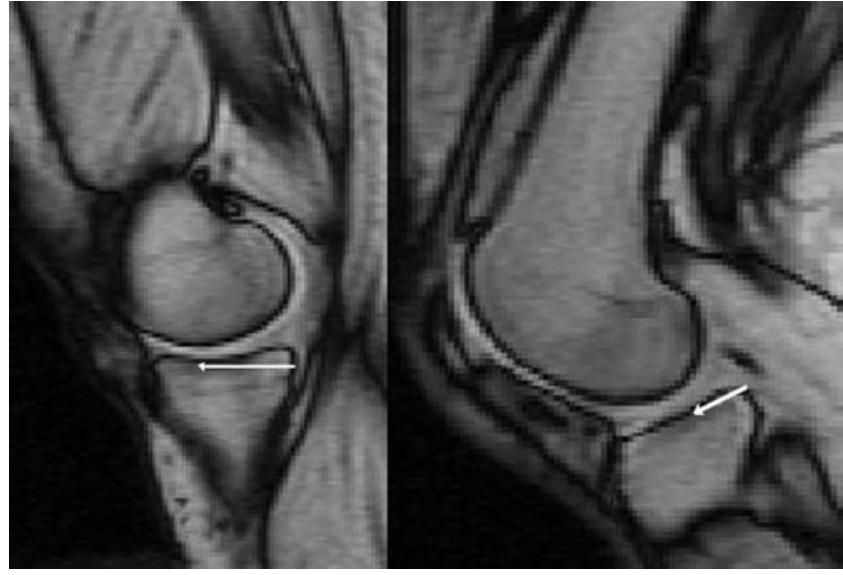

Fig. 1

Sagittal images through the centre of the medial compartment at $0^{\circ}$ flex ion, and through the centre of the lateral compartment at $45^{\circ}$, demonstrating the measurement of tibiofemoral contact from the posterior tibial cortex.

validated for use in patients with ACL-injured and reconstructed knees. ${ }^{24}$

Tibiofemoral contact point measurement from MRI scans. Imaging of both knees was performed using a 1.5T Siemens Magnetron Vision (Erlangen, Germany), using a body coil to generate parasagittal images perpendicular to each tibial plateau. Patients were scanned using a spoiled gradient echo sequence. ${ }^{25}$ A total of 16 slices was generated (eight through each knee) approx $10 \mathrm{~mm}$ apart (TR = 160.0; TE = $2.3 / 1 ; \mathrm{TA}=00: 46)$, with a $256 \times 256$ matrix. Scans took 38 seconds each, with a complete session time of 35 minutes. These images were directly downloaded as Dicom format files and were analysed using Osiris software version 4.11 (Université de Geneva, Geneva, Switzerland).

In order to study the kinematic effects of ACL injury, the patients performed a supine leg-press between $0^{\circ}$ and $90^{\circ}$ knee flexion. The flexed position was maintained using a customised wooden sled, with a sliding footplate arrested by dowel pins at $15^{\circ}$ intervals. Patients were scanned at each position twice, once while relaxed, and once pressing down against a footplate weighted with a sandbag imposing a force in line with the axial skeleton of $150 \mathrm{~N}$.

From the sagittal images which were generated, those representing the centre of the medial and lateral compartments of the knee, respectively, were chosen. The position of the tibiofemoral contact was recorded as the distance from the posterior tibial cortex to the point of tibiofemoral contact for both the medial and lateral femoral condyles, respectively ${ }^{20}$ (Fig. 1). The reliability of this method of measurement has been tested for accuracy and consistent selection of images representing the centre of the medial and lateral compartment. This method has strong reliability. Tested for the observer who performed the measurements, there was an intraclass correlation of 0.94 which is significant at a $99 \%$ confidence interval (CI 0.91 to 0.97 ). 


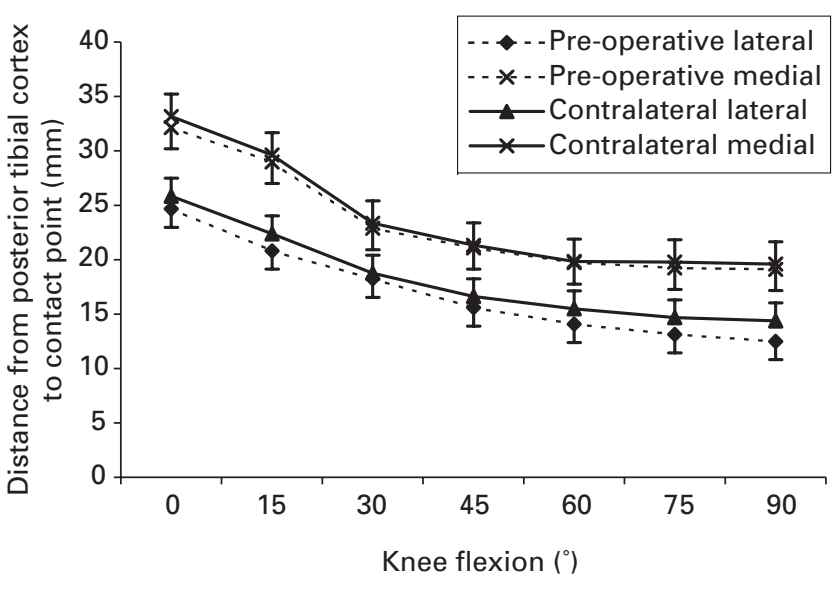

Fig. 2a

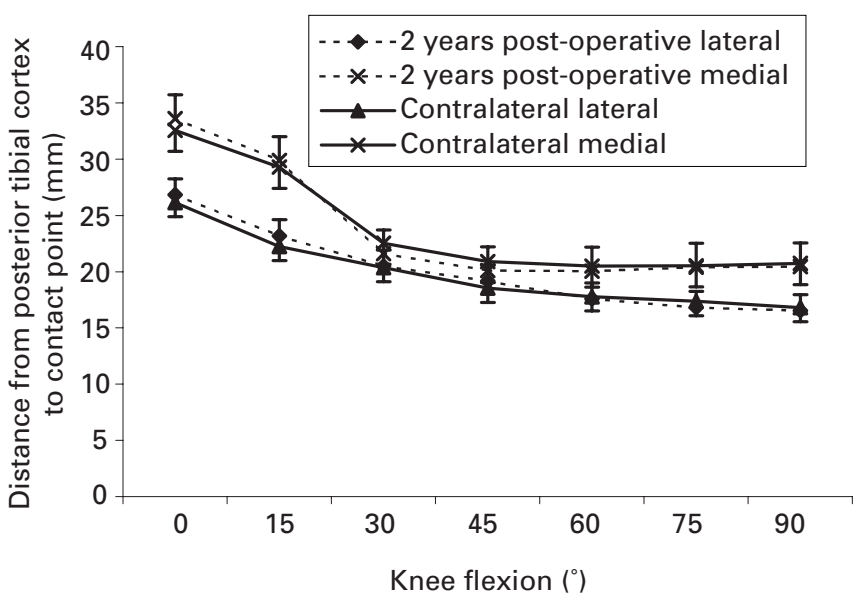

Fig. 2c

Statistical analysis. Tibiofemoral contact patterns of injured and healthy contralateral knees were compared using a repeated measures ANOVA model. In order to analyse the effects of surgery on the contact patterns the injured knees were compared with the contralateral knees pre-operatively and at 12 weeks and two years following reconstruction. In order to analyse the effects of time on the contact patterns, those of the injured knees were compared longitudinally: pre-operative, 12 weeks and two years post-operatively; and the contact patterns of the contralateral knees were compared at the same three time points. The data recorded for 12 healthy control subjects were included for analysis of changes in the healthy knee over time. Where differences in kinematics were found, a Bonferroni comparison was used for post hoc analysis. The significance level was set at $\mathrm{p}=$ 0.05. Data analysis was performed on SPSS version 11.5 (SPSS Inc., Chicago, Illinois).

\section{Results}

Surgical outcomes. With $134 \mathrm{~N}$ anterior drawer, the KT 1000 showed a mean side-to-side difference in passive an-

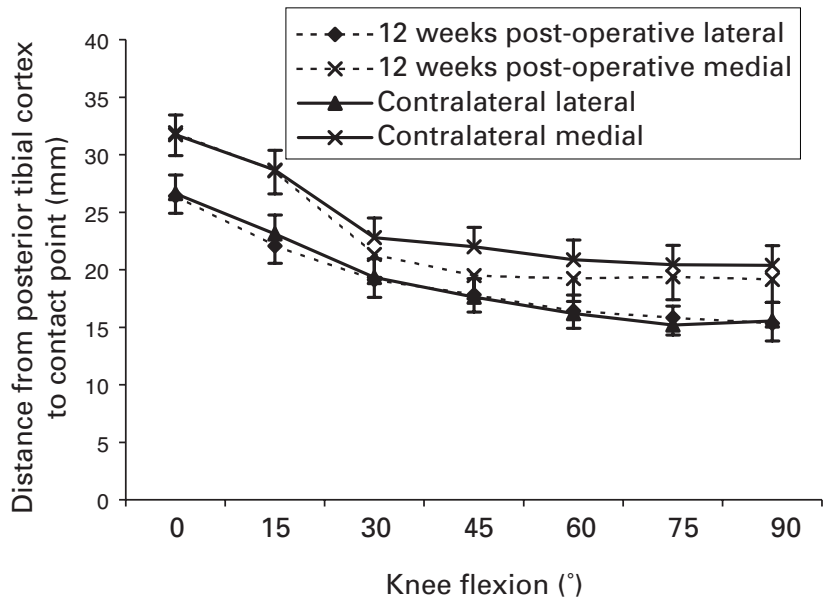

Fig. $2 b$

Tibiofemoral contact patterns in the injured and healthy contralateral knees (mean SE). Figure 2a - Pre-operatively contact patterns occurred more posteriorly in the injured knees $(p=0.014)$. Figure $2 b-A t 12$ weeks there was no significant difference between the reconstructed and healthy contralateral knee $(p=0.117)$. Figure $2 c-A t$ two years, there was no significant difference between the reconstructed and healthy contralateral knee $(p=0.909)$.

terior laxity pre-operatively of $3.9 \mathrm{~mm}$ (SD $2 ; 0$ to 8 ), at 12 weeks of $2.2 \mathrm{~mm}$ (SD $2 ;-1$ to 7 ) and at two years of $2.1 \mathrm{~mm}$ (SD $1.8 ;-1$ to 4.5 ). At two years post-operatively, there were 11 patients whose anterior laxity was graded 'excellent' $(<3 \mathrm{~mm}$ ) and seven graded 'good' ( 3 to $5 \mathrm{~mm}$ side-to-side difference) according to the Cincinnati knee rating. No patient was graded fair or poor. On manual maximum anterior drawer the KT 1000 showed a mean side-to-side difference pre-operatively of $5.1 \mathrm{~mm}$ (SD 2.6; 0 to 9.8), at 12 weeks of $2.5 \mathrm{~mm}$ (SD 2.2;-1 to 6.2) and at two years of 2.1 $\mathrm{mm}$ (SD 2.3; 0 to 7.6 ).

The mean pre-operative Cincinnati score was 57 (SD 11) of 100 . At 12 weeks post-operatively, the mean score was 76 (SD 10), at two years post-operatively 90 (SD 11). Of the 18 patients in the study at two years the complications included one with deep-vein thrombosis and one with medial compartment narrowing on radiograph. While there were no professional players in this group, all those playing amateur sports had returned to their preferred sport.

Tibiofemoral contact patterns in healthy contralateral knees. The tibiofemoral contact pattern of the healthy con- 


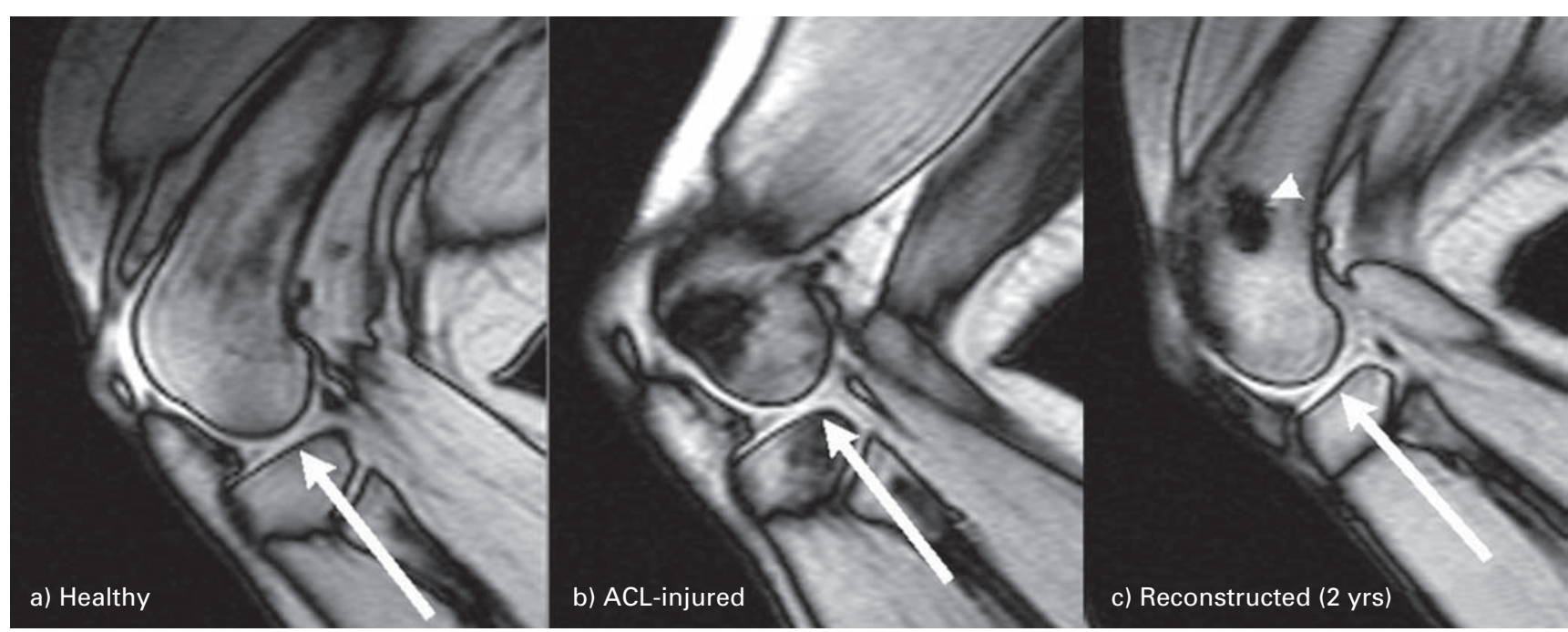

Fig. 3

Sagittal MRI of the lateral compartments of the a) healthy contralateral, b) ACL-injured, and c) reconstructed knee at two years, of a subject performing a loaded leg-press against $150 \mathrm{~N}$. The tibiofemoral contact point in the ACL-injured knee is more posterior on the tibial plateau, and is normalised by reconstruction (large arrows). The small arrow shows an artefact on the MRI caused by the femoral pin.

tralateral knees differed between the medial and lateral compartments $\left(\mathrm{F}_{(1,156)}=321.6 ; \mathrm{p}<0.001\right)$. From $0^{\circ}$ to $30^{\circ}$ knee flexion, the medial femoral condyle contact pattern moved posteriorly on the tibial plateau from a position at $0^{\circ}$ that was a mean of $33 \mathrm{~mm}$ (SD 3.9) from the posterior tibial cortex to a position at $30^{\circ}$ that was a mean of $23.3 \mathrm{~mm}$ (SD 3.3). Between $45^{\circ}$ and $90^{\circ}$, posterior movement was minimal (mean $0.4 \mathrm{~mm}$ ), the medial femoral condyle remaining centred on the tibial plateau.

The lateral femoral condyle traced a contact pattern through more posterior excursion than the medial. Between $0^{\circ}$ and $90^{\circ}$, the pattern of tibiofemoral contact moved back steadily across the tibial plateau from a mean of $25.8 \mathrm{~mm}$ (SD 3.1) from the posterior tibial cortex to a mean of 14.4 $\mathrm{mm}$ (SD 2.7) at $90^{\circ}$ knee flexion.

Tibiofemoral contact patterns in ACL-injured knees. The injured knee had a similar contact pattern to the healthy contralateral knee, but it occurred more posteriorly on the tibial plateau $\left(\mathrm{F}_{(1,152)}=6.2 ; \mathrm{p}=0.014\right)$ (Fig. 2). The medial compartment was similar to the healthy contralateral knee; at $0^{\circ}$ knee flexion the tibiofemoral contact occurred anteriorly on the tibial plateau by a mean of $32.5 \mathrm{~mm}$ (SD 5.0) from the posterior tibial cortex, and contact moved back to the centre of the tibial plateau to a mean of $22.8 \mathrm{~mm}$ (SD 2.9) from the posterior tibial cortex at $30^{\circ}$ knee flexion. Between $30^{\circ}$ and $90^{\circ}$ flexion, the contact pattern moved back a further mean of $2.7 \mathrm{~mm}$, so that at $90^{\circ}$ flexion it was positioned a mean of $19.1 \mathrm{~mm}$ (SD 2.3) from the posterior tibial cortex.

The lateral contact pattern translated posteriorly on the tibial plateau further than the medial contact pattern. At $0^{\circ}$ the lateral contact point was positioned a mean of $24.7 \mathrm{~mm}$
(SD 4.3) from the posterior tibial cortex, and translated posteriorly to finish a mean of $12.5 \mathrm{~mm}$ (SD 2.8) from the posterior tibial cortex at $90^{\circ}$.

The medial and lateral compartments had different contact patterns in injured and healthy contralateral knees, demonstrating longitudinal rotation of the knee $\left(\mathrm{F}_{(1,136)}=\right.$ $315.42 ; \mathrm{p}<0.001)$. While the mean difference in the tibiofemoral contact points between the injured and healthy knee was $0.5 \mathrm{~mm}$ for the medial compartment and $1.3 \mathrm{~mm}$ for the lateral compartment, this was not significant $\left(\mathrm{F}_{(1,152)}\right.$ $=1.2 ; \mathrm{p}=0.27$ ).

At 12 weeks after surgery, the tibiofemoral contact pattern in the ACL-reconstructed knees was no longer different from the contralateral healthy knees $\left(\mathrm{F}_{(1,152)}=2.5 ; \mathrm{p}=\right.$ $0.117)$. Likewise, at two years after reconstruction the tibiofemoral contact pattern in the reconstructed knees was the same as that of the contralateral knees $\left(\mathrm{F}_{(1,136)}=0.013\right.$; $\mathrm{p}=0.909$ ) (Fig. 3).

Performing a leg-press did not produce a different tibiofemoral contact pattern to the relaxed contact pattern, in either the injured or healthy knees $\left(\mathrm{F}_{(1,136)}=0.76 ; \mathrm{p}=\right.$ $0.386)$. Similarly, at 12 weeks post-operatively, there was no difference between the loaded and unloaded contact patterns $\left(\mathrm{F}_{(1,152)}=1.3 ; \mathrm{p}=0.256\right)$ nor at two years $\left(\mathrm{F}_{(1,136)}=\right.$ $1.3 ; \mathrm{p}=0.256$ ).

Changes in tibiofemoral contact patterns to healthy knees over time. There was a significant difference in the contact pattern of the healthy contralateral knees at the three time points, pre-operatively, at 12 weeks and two years after reconstruction $\left(\mathrm{F}_{(1,204)}=11.5 ; \mathrm{p}<0.001\right)$. Post hoc tests showed the difference to be between 12 weeks and two years in the healthy contralateral knees $(\mathrm{p}=0.003)$. The lat- 

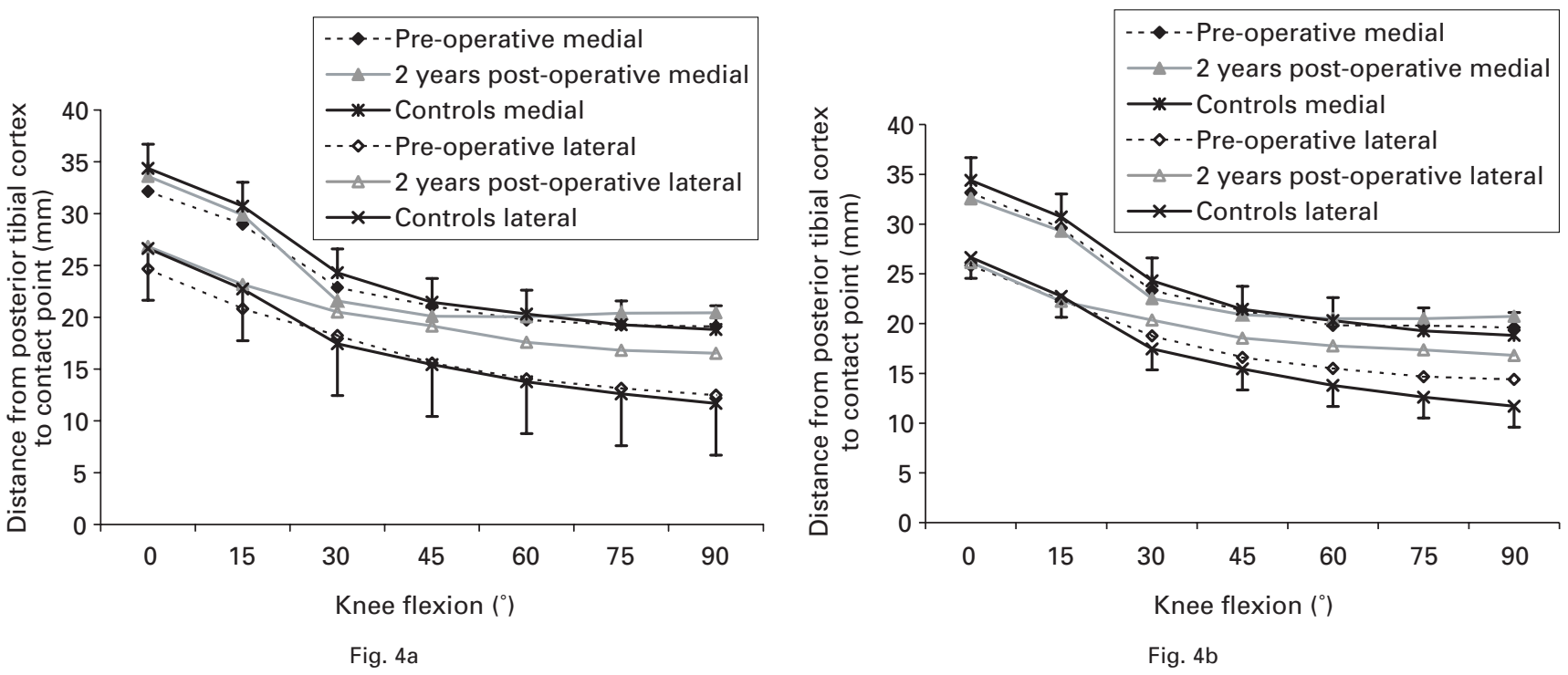

Changes over time in a) ACL-reconstructed knees and control subjects (mean, SE) and b) the healthy contralateral knees and control subjects. Changes occurred in both the ACL-reconstructed and healthy knees; posterior translation of contact points in the lateral compartment decreased. In this figure healthy control subjects are included for comparison with the ACL-reconstructed subjects.

eral compartment contact pattern was most affected by the changes over time $\left(\mathrm{F}_{(2,204)}=42.5 ; \mathrm{p}=0.021\right)$ (Fig. 4).

The pre-operative contact pattern in the lateral compartment of healthy contralateral knees was similar to the contact pattern at two years at $0^{\circ}$ and $15^{\circ}$ knee flexion. However, from $45^{\circ}$ to $90^{\circ}$ knee flexion, the lateral tibiofemoral contact pattern did not translate as far posteriorly on the tibial plateau as it had at pre-operative testing. Preoperatively, the healthy knee contact position at $45^{\circ} \mathrm{knee}$ flexion was a mean of $16.0 \mathrm{~mm}$ (SD 2.0) and at two years was a mean of $18.5 \mathrm{~mm}$ (SD 2.3) from the posterior tibial cortex reference point. At $90^{\circ} \mathrm{knee}$ flexion, the pre-operative position was a mean of $14.4 \mathrm{~mm}$ (SD 2.8) and at two years was a mean of $16.8 \mathrm{~mm}$ (SD 2.3) from the posterior tibial cortex. At two years, the lateral tibiofemoral contact pattern did not translate as far posteriorly on the tibial plateau as it had pre-operatively, but the contact pattern of the medial femoral condyle was unchanged. This indicates that the knees had lost some of the longitudinal rotation evident during knee flexion at the pre-operative measurement.

Changes in tibiofemoral contact patterns to reconstructed knees over time. Changes over time were also evident in the reconstructed knees. Post hoc analysis showed a difference in the contact pattern pre-operatively and at two years post-operatively in the reconstructed knees $(\mathrm{p}<0.01)$. The medial compartment contact pattern was unchanged but the lateral tibiofemoral contact pattern did not translate as far posteriorly over the two years of the study. Pre-operatively the tibiofemoral contact pattern moved back to a mean of $12.5 \mathrm{~mm}$ (SD 2.8) from the posterior tibial cortex at $90^{\circ}$ knee flexion and at two years the tibiofemoral contact pat- tern moved back to a mean of $16.5 \mathrm{~mm}$ (SD 2.5) from the posterior tibial cortex.

\section{Discussion}

The reconstruction was successful for all 20 subjects at 12 weeks and in the 18 patients followed for two years according to instrumented laxity tests and the Cincinnati rating system. The mean passive anterior laxity tested with manual maximum anterior force at two years was $2.1 \mathrm{~mm}$ (SD 2.3), which compares with $2 \mathrm{~mm}$ (SD 1.5) reported by Shelbourne and $\mathrm{Gray}^{26}$ and is similar to other studies. 2,3,27,28 Likewise the Cincinnati scores for this study compare favourably with previous reports. A group of 250 patients who underwent ACL had a mean score of 56 (SD 9) preoperatively and 89 (SD 11) at two years' follow-up ${ }^{24}$ while of a group of 44 patients with patellar tendon reconstructions, 32 had scores of more than 86 of $100 .^{29}$ The instrumented and functional outcomes indicate good recovery of the patients in the study.

The ACL-injured knees pre-operatively had a more posterior tibiofemoral contact pattern than the healthy contralateral knees. This shows that anterior laxity of the knee reported in vitro and measured in vivo by passive arthrometry, ${ }^{23}$ is present during a leg-press and expressed in the altered contact pattern. Altered kinematics in the sagittal plane have also been described during walking ${ }^{30}$ and stepup activities. ${ }^{17,31}$ The difference in the tibiofemoral contact pattern pre-operatively provided a baseline from which to measure the effect of reconstructive surgery on the kinematics of the knee.

Rotary instability of the ACL-injured knees, as reported in vitro ${ }^{32}$ and in vivo, ${ }^{33}$ could be expected to produce a dif- 
ference in the medial and lateral contact patterns. This difference reflects rotation of the knee during flexion. ${ }^{20,34} \mathrm{In}$ the present study the difference was more pronounced in the lateral than the medial compartments of the ACLinjured knees, although this failed to reach significance. Examples of the dynamic rotary instability have been reported in other activities, including a step-up activity ${ }^{17}$ and during the swing phase of gait in patients with ACL injuries; ${ }^{15}$ this was reported as internal tibial rotation. As the tibia internally rotates, the femoral condyle moves posteriorly on the tibial plateau, so our finding of a posterior tibiofemoral contact pattern in the lateral compartment could reflect this.

At 12 weeks after reconstruction the tibiofemoral contact pattern, and thus the kinematics of the operated knee, was the same as that of the contralateral healthy knee. There are no other studies that have examined kinematics at 12 weeks after surgery. However, a gait analysis study at three weeks after ligament reconstruction has shown decreased flexion/extension movement during the swing phase and decreased knee flexion during the stance phase, ${ }^{13}$ which had returned to normal six months following surgery. Abnormal kinematics three weeks after surgery may be because healing is incomplete.

The tibiofemoral contact pattern of the reconstructed knees at two years was also the same as the healthy contralateral knees, indicating that the kinematics, as assessed by this method, had been restored to normal. The MRI method that we used demonstrated a difference in contact pattern between the injured and the healthy knees pre-operatively but this difference was no longer present at two years. The persistant asymmetry between medial and lateral tibiofemoral contact patterns reflects the fact that longitudinal rotation occurs during knee flexion. These aspects of knee kinematics are consistent with the patterns of healthy control subjects. ${ }^{20}$

Other studies have not found that knee kinematics were restored by reconstruction. ${ }^{8,9,16}$ In a one-year follow-up of bone-patellar tendon-bone reconstruction using an RSA technique, the tibial rotation and femoral translation still exhibited the same abnormal characteristics that had been present pre-operatively. In an open field MRI, Logan et $\mathrm{al}^{7}$ found persistent anterior subluxation of the lateral tibial plateau on squatting. Tashman et $\mathrm{al}^{8}$ found increased external rotation of the tibia relative to the femur during running. Four of these six patients also had increased lateral tibial translation, although this was not significant. ${ }^{8}$ In a gait analysis study, anterior translation of the tibial tubercle was greater six months following functionally successful reconstructive surgery. ${ }^{16}$ It may be that at six months sensorimotor re-education and rehabilitation were not yet complete. ${ }^{35}$ While advances in technology and imaging now enable accurate and direct measurement of knee kinematics, there is still much to understand since altered kinematics in reconstructed knees may have implications for long-term degenerative sequelae.
Changes to the healthy knee over the two-year period of the study are interesting. No other studies have examined kinematics of the healthy contralateral knees in the postoperative analysis. There is a suggestion that the kinematics of healthy contralateral knees of ACL-injured subjects are not the same as normal control subjects, implying that ACL-injured subjects have some intrinsic kinematic anomaly. ${ }^{36}$ However, this is the first reported longitudinal study of the healthy knees of ACL-injured or reconstructed patients which examines the long-term changes to the healthy knees.

The loss of posterior translation of the contact point in the lateral compartment of healthy contralateral knees over the two-year period appears similar to the loss of contact point translation in the lateral compartment seen in knees with early osteoarthritis. ${ }^{37}$ However, there were no symptoms or MRI evidence of osteoarthritis in these healthy contralateral knees. A further study of these knees over the long-term may clarify whether these changes are indeed indicative of early osteoarthritis.

Anterior cruciate ligament reconstruction by hamstring autograft restored the tibiofemoral contact pattern to that of the healthy contralateral knee. Asymmetrical contact patterns between the medial and lateral compartments of the knee indicated that longitudinal rotation occurs during flexion. Loading at $150 \mathrm{~N}$ did not alter the contact pattern of the knee. These kinematic characteristics are consistent with those of the healthy knee. However, it does appear that the kinematics of the ACL-reconstructed and the healthy contralateral knee may change with time.

\section{Supplementary Material}

$\ddot{e}$ A further opinion by Mr Andrew Williams is available with the electronic version of this article on our website at www.jbjs.org.uk

This research was supported by a grant from the National Health and Medical Research Council of Australia, and the first author was a recipient of an NHMRC Biomedical scholarship. The research team thanks Dr Bruce Shadbolt (PhD) for his statistics expertise, Ms Dianne Lane, MRI radiographer and Drs Michae Gillespie and Rob Creer for recruiting patients and their surgical skills. Many thanks to the subjects who volunteered their time and confidence.

No benefits in any form have been received or will be received from a commercial party related directly or indirectly to the subject of this article.

\section{References}

1. Jomha N, Borton D, Clingeleffer A, Pinczewski L. Long term osteoarthritic changes in anterior cruciate ligament reconstructed knees. Clin Orthop 1999;358: 188-93.

2. $\mathbf{0}$ 'Neill D. Arthroscopically assisted reconstruction of the anterior cruciate ligament: a follow-up report. J Bone Joint Surg [Am]2001;83-A:1329-32.

3. Pinczewski L, Deehan M, Salmon L, Russell V, Clingeleffer A. A five-year comparison of patella tendon versus four-strand hamstring tendon autograft for arthroscopic reconstruction of the anterior cruciate ligament. Am J Sports Med 2002;30: 523-36.

4. Gillquist J. Repair and reconstruction of the ACL: is it good enough? Arthroscopy 1993;9:68-71

5. Kanisawa I, Banks A, Banks S, Moriya H, Tsuchiya A. Weight-bearing knee kinematics in subjects with two types of anterior cruciate ligament reconstructions. Knee Surg Sports Traumatol Arthrosc 2003;11:16-22.

6. Mahfouz M, Traina S, Komistek R, Dennis D. In vivo determination of knee kinematics in patients with hamstring or patella tendon ACL grafts. J Knee Surg 2003;16: 197-202. 
7. Logan M, Williams A, Lavelle J, Gedroyc W, Freeman M. Tibiofemoral kinematics following successful anterior cruciate ligament reconstruction using dynamic multiple resonance imaging. Am J Sports Med 2004;32:984-92.

8. Tashman S, Collon D, Anderson K, Kolowich P, Anderst W. Abnormal rotational motion during running after anterior cruciate ligament reconstruction. Am J Sports Med 2004;32:975-83

9. Brandsson S, Karlsson J, Sward L, et al. Kinematics of the knee after anterior cruciate ligament reconstruction. Am J Sports Med 2002;30:361-7.

10. Yagi M, Wong $\mathbf{E}$, Kanamori $\mathbf{A}$, et al. Biomechanical analysis of an anatomic anterior cruciate ligament reconstruction. Am J Sports Med 2002;30:660-6.

11. Papergeorgiou C, Gil J, Kanamori A, et al. The biomechanical interdependence between the anterior cruciate ligament replacement graft and the medial meniscus. Am J Sports Med 2001;29:226-31.

12. Woo S, Kanamori A, Zeminski J, et al. The effectiveness of reconstruction of the anterior cruciate ligament with hamstrings and patella tendon: a cadaveric study comparing anterior tibial and rotational loads. J Bone Joint Surg [Am] 2002;84-A: 907-14.

13. De Vita P, Hortobagyi T, Barrier J. Gait biomechanics are not normal after anterior cruciate ligament reconstruction and accelerated rehabilitation. Med Sci Sports Exerc 1998;30:1481-8

14. Bulgheroni P, Bulgeroni M, Andrini L, Guffanti P, Giughello A. Gait patterns after anterior cruciate ligament reconstruction. Knee Surg Sports Traumatol Arthrosc 1997:5:14-21.

15. Georgoulis A, Papadonikolakis A, Papageorgiou C, Mitsou A, Stergiou N. Three-dimensional tibiofemoral kinematics of the anterior cruciate ligament deficient and reconstructed knee during walking. Am J Sports Med 2003;31:75-9.

16. Beard D, Murray D, Gill H, et al. Reconstruction does not reduce tibial translation in the cruciate-deficient knee: an in vivo study. J Bone Joint Surg [Br]2001;83-B:1098-103.

17. Brandsson S, Karlsson J, Eriksson B, Karrholm J. Kinematics after tear in the anterior cruciate ligament: dynamic bilateral radiostereometric studies in 11 patients. Acta Orthop Scand 2001;72:372-8.

18. Scarvell J, Smith P, Refshauge K, Galloway H, Woods K. Comparison of kinematics in the healthy and ACL injured knee using MRI. J Biomech 2005;38:255-62.

19. Scarvell J, Smith P, Refshauge K, Galloway H, Woods K. Kinematic consequences of chronic ACL deficiency. J Bone Joint Surg [Br]2003;86-B(Suppl IV):479-80.

20. Scarvell J, Smith P, Refshauge K, Galloway H, Woods K. Evaluation of a method to map tibiofemoral contact points using MRI. J Orthop Res 2004;22:788-93.

21. Beynnon B, Johnson R, Fleming B. The science of anterior cruciate ligament rehabilitation. Clin Orthop 2002;402:9-20.

22. Shelbourne K, Nitz $\mathbf{P}$. Accelerated rehabilitation after anterior cruciate ligament reconstruction. Am J Sports Med 1990;18:292-8.
23. Daniel D, Malcolm L, Losse G. Instrumented measurement of anterior laxity of the knee. J Bone Joint Surg [Am] 1985;67-A:720-6.

24. Barber-Westin S, Noyes F, McCloskey J. Rigorous statistical reliability, validity, and responsiveness testing of the Cincinnati knee rating system in 350 subjects with uninjured, injured, or anterior cruciate ligament reconstructed knees. Am J Sports Med 1999;27:402-16

25. Behrens J, King M. Application highlights of Vb33a Software. Siemens Medical Engineering 2000:2-3

26. Shelbourne K, Gray T. Anterior cruciate ligament reconstruction with autogenous patellar tendon graft in patients with articular cartilage damage: a two- to nin-year follow-up. Am J Sports Med 1997;25:786-95.

27. Shaieb M, Kan D, Chang S, Marumoto J, Richardson A. A prospective randomised comparison of patella tendon versus semitendinosus and gracilis tendon autografts for anterior cruciate ligament reconstruction. Am J Sports Med 2002;30: 214-20

28. Barber-Westin S, Noyes F, Heckman T, Shaffer B. The effect of exercise and rehabilitation on the antero-posterior knee displacements after anterior cruciate ligament autograft reconstruction. Am J Sports Med 1999;27:84-93.

29. Hrubesch R, Rangger C, Reichkendler M, et al. Comparison of score evaluations and instrumented measurement after anterior cruciate ligament reconstruction. $\mathrm{Am} \mathrm{J}$ Sports Med 2000;28:850-6.

30. Dennis D, Komistek R, Hoff W, Gabriel S. In vivo knee kinematics derived using an inverse perspective technique. Clin Orthop 1996;331:107-17.

31. Vergis A, Hindriks $\mathbf{M}$, Gillquist $\mathbf{J}$. Sagittal plane translations of the knee in anterior cruciate deficient subjects and controls. Med Sci Sports Exerc 1997;29:1561-6.

32. Sakane M, Livesay G, Fox R, et al. Relative contribution of the anterior cruciate ligament, medial collateral ligament and bony contact to the anterior stability of the knee. Knee Surg Sports Traumatol Arthrosc 1999;7:93-7.

33. Rudolph K, Axe M, Buchanan T, Scholz J, Snyder-Mackler L. Dynamic stability in the anterior cruciate ligament deficient knee. Knee Surg Sports Traumatol Arthrosc 2001;9:62-71.

34. Hill P, Iwaki H, Pinskerova V, Freeman M. Does femoral roll-back occur in knee flexion?: a MRI study. J Bone Joint Surg [Br]2000;82-B(Suppl 1):43.

35. Neitzel JA, Kernozek TW, Davies GJ. Loading response following anterior cruciate ligament reconstruction during the parallel squat exercise. Clin Biomech (Bristol, Avon) 2002;17:551-4.

36. Andriacchi T, Birac D. Functional testing in the anterior cruciate deficient knee. Clin Orthop 1993;288:40-7.

37. Saari T, Carlsson L, Karlsson J, Karrholm J. Knee kinematics in medial arthrosis: dynamic radiostereometry during active extension and weight-bearing. J Biomech 2005;38:258-92. 